\title{
Expression of Mesenchymal Stem Cells-Related Genes and Plasticity of Aspirated Follicular Cells Obtained from Infertile Women
}

\author{
Edo Dzafic, ${ }^{1}$ Martin Stimpfel, ${ }^{1}$ Srdjan Novakovic, ${ }^{2}$ \\ Petra Cerkovnik, ${ }^{2}$ and Irma Virant-Klun ${ }^{1}$ \\ ${ }^{1}$ Department of Obstetrics and Gynaecology, University Medical Centre Ljubljana, Šlajmerjeva 3, 1000 Ljubljana, Slovenia \\ ${ }^{2}$ Department of Molecular Diagnostics, Institute of Oncology Ljubljana, Zaloška 2, 1000 Ljubljana, Slovenia
}

Correspondence should be addressed to Irma Virant-Klun; irma.virant@kclj.si

Received 23 November 2013; Revised 21 January 2014; Accepted 22 January 2014; Published 3 March 2014

Academic Editor: Jeroen Krijgsveld

Copyright (C) 2014 Edo Dzafic et al. This is an open access article distributed under the Creative Commons Attribution License, which permits unrestricted use, distribution, and reproduction in any medium, provided the original work is properly cited.

\begin{abstract}
After removal of oocytes for in vitro fertilization, follicular aspirates which are rich in somatic follicular cells are discarded in daily medical practice. However, there is some evidence that less differentiated cells with stem cell characteristics are present among aspirated follicular cells (AFCs). The aim of this study was to culture AFCs in vitro and to analyze their gene expression profile. Using the RT ${ }^{2}$ Profiler PCR array, we investigated the expression profile of 84 genes related to stemness, mesenchymal stem cells (MCSs), and cell differentiation in AFCs enriched by hypoosmotic protocol from follicular aspirates of infertile women involved in assisted reproduction programme in comparison with bone marrow-derived mesenchymal stem cells (BM-MSCs) and fibroblasts. Altogether the expression of 57 genes was detected in AFCs: 16 genes (OCT4, CD49f, CD106, CD146, CD45, CD54, IL10, IL1B, TNF, VEGF, VWF, HDAC1, MITF, RUNX2, PPARG, and PCAF) were upregulated and 20 genes (FGF2, CASP3, CD105, CD13, CD340, CD73, CD90, KDR, PDGFRB, BDNF, COL1A1, IL6, MMP2, NES, NUDT6, BMP6, SMURF2, BMP4, GDF5, and JAG1) were downregulated in AFCs when compared with BM-MSCs. The genes which were upregulated in AFCs were mostly related to MSCs and connected with ovarian function, and differed from those in fibroblasts. The cultured AFCs with predominating granulosa cells were successfully in vitro differentiated into adipogenic-, osteogenic-, and pancreatic-like cells. The upregulation of some MSC-specific genes and in vitro differentiation into other types of cells indicated a subpopulation of AFCs with specific stemness, which was not similar to those of BM-MSCs or fibroblasts.
\end{abstract}

\section{Introduction}

In infertile women, oocytes are retrieved by ultrasoundguided transvaginal follicular aspiration in the assisted reproduction programme. After removal of oocytes for in vitro fertilization, follicular aspirates which are rich in somatic follicular cells are discarded in daily medical practice. Each follicular aspirate consists of numerous types of somatic cells along with follicular fluid [1]. The main types of aspirated follicular cells (AFCs) are represented by granulosa cells (GCs) and theca cells (TCs). The main role of GCs is to support the oocyte by providing some nutrients that are essential for oocyte growth and development and to accumulate the metabolites secreted by the oocyte. On the other hand, TCs produce androgens which are converted to estradiol by GCs [2]. Nevertheless, the follicular aspirate is also composed of other types of cells such as red and white blood cells thus reflecting good vascularization and some resident immune cells in ovarian follicles. Moreover, also some vaginal and ovarian surface epithelial cells can be present among AFCs since these tissues are penetrated during transvaginal follicular aspiration $[3,4]$.

Follicular aspirates are discarded in daily medical practice but could be an important source for potential research, diagnostics (e.g., immunoassays), and cell therapy in the future, since it has already been evidenced that subpopulations of AFCs can express some stem cell characteristics [5]. Especially, GCs represent a very interesting subpopulation of AFCs as demonstrated by several studies and recently reviewed by our group [6]. GCs originate from 
ovarian surface epithelium and form the major part of the growing follicle, possess a remarkable proliferation activity, and represent a predominant type of AFCs [7]. Studies evidenced expression of the stemness-related marker OCT4 and multiple mesenchymal linage-related markers in GCs along with their differentiation into other types of cells [8], especially spontaneous differentiation into osteogeniclike cells [9]. Moreover, the possible contribution of less differentiated GCs in development of ovarian cancers has been suggested [10]. Along with GCs, it has also been shown that subpopulation of TCs contains putative stem cells [11].

It is of great scientific interest to isolate, proliferate, and research the less differentiated/progenitor cells among AFCs for potential medical use in the future. However, there have been no studies until now which would analyze the broader gene expression profile of AFCs and elucidate the potential relation of AFCs to mesenchymal stem cells (MSCs), the most common cells tested in the regeneration of impaired ovarian function in the animal models $[12,13]$.

The aim of this study was therefore to analyse the expression of eighty-four different genes related to stemness (pluripotency), MSCs, and cell differentiation in cultured AFCs from follicular aspirates of infertile women included in the assisted reproduction programme in comparison with bone marrow-derived MSCs (BM-MSCs) and human dermal fibroblasts (HDFs). We also tested the osteogenic, adipogenic, and pancreatic differentiation in cultured AFCs to evidence their plasticity. Our results showed that cultured AFCs expressed specific stemness related to MSCs but other than in BM-MSCs and somatic fibroblasts. Moreover, the cultured AFCs were able to differentiate into adipogenic-, osteogenic-, and pancreatic-like cells in vitro.

\section{Materials and Methods}

2.1. Collection of AFCs. This study was approved by the Slovenian Medical Ethical Committee (Ministry of Health, number 196/10/07). After written informed consents, follicular aspirates were collected by transvaginal ultrasound-guided aspiration from twelve infertile patients treated with controlled ovarian hyperstimulation for assisted reproduction. Patients were treated with various exogenous gonadotropins as described previously [14]. After removal of the cumulus oophorus-oocyte-complexes, the AFCs were enriched from the follicular aspirates using hypoosmotic technique as described by Lobb and Younglai [15], mainly to remove red blood cells. Briefly, the freshly collected follicular aspirates from each patient were pooled in conical bottomed $50 \mathrm{~mL}$ polypropylene centrifuge tubes and centrifuged at $1400 \mathrm{rpm}$ for $6 \mathrm{~min}$. The supernatant was aspirated and the remaining cell slurry was pipetted into a $15 \mathrm{~mL}$ conical bottomed polystyrene centrifuge tube. To the cell slurry $9.0 \mathrm{~mL}$ of sterile distilled water was added and the tube was capped and mixed. After $60 \mathrm{~s}, 1.0 \mathrm{~mL}$ of $10 \mathrm{x}$ concentrated phosphate buffer saline (PBS; pH 7.4) was added and the tube was capped and mixed. The tubes were then centrifuged at $800 \mathrm{rpm}$ for $3 \mathrm{~min}$; the supernatant was discarded; the cell pellet was resuspended in
$0.5 \mathrm{~mL}$ of culture medium and transferred into a culture dish. From each patient, one AFCs culture was established.

2.2. Cell Cultures. Cells were cultured in gelatin-coated 4-well culture dish ( $15 \mathrm{~mm}$ well diameter) at concentration of $1 \times 10^{5}$ cells per well. For the culture medium, DMEM/F12 (Sigma-Aldrich) with 20\% follicular fluid serum (FF) retrieved from the in vitro fertilization programme was used. FF was prepared as described previously by Stimpfel et al. [16]. The cells were cultured in a $\mathrm{CO}_{2}$ incubator at $37^{\circ} \mathrm{C}$ and $6 \% \mathrm{CO}_{2}$ in air and daily monitored at the heat-staged inverted microscope (Nikon, Japan). When the cell culture was set up, the culture medium was replaced by a fresh medium on the next day to remove the remaining red blood cells. The cell splitting was performed when needed using $0.15 \%$ trypsin (Sigma-Aldrich). Alive AFCs were maintained in a cell culture based on two criteria: (i) cells were attached to the surface of culture dish and (ii) cells proliferated. The cells were cultured up to 2 months.

2.3. Gene Expression Analysis. Human Mesenchymal Stem Cell RT ${ }^{2}$ Profiler PCR Array (PAHS-082, SABiosciences, Qiagen) was used to evaluate the expression of 84 specific genes related to stemness (pluripotency), MSCs, and cell differentiation-osteogenesis, adipogenesis, chondrogenesis, myogenesis, and tenogenesis (see Supplementary Table 1 available online at http://dx.doi.org/10.1155/2014/508216). After 5 days of culturing, three AFCs cultures from three different patients who aged 36 years (uterine abnormality), 36 years (no indication of infertility/male infertility), and 38 years (tubal factor of infertility) were pooled together and analysed along with control samples. As a positive control, a commercially available cell line of bone marrow-derived mesenchymal stem cells (BM-MSCs) was used (Chemicon, Millipore, cat. number SCC034). These cells were cultured in a mesenchymal stem cell expansion medium provided by the same producer (cat. number SCM015). As a negative control, adult human dermal fibroblasts (HDFs) were used (Cascade Biologics, Invitrogen, cat. number C-013-5C), which were cultured in DMEM/F12 (Sigma-Aldrich) with 10\% FBS (Gibco, Invitrogen).

The total RNA was isolated from $10^{5}$ to $10^{6}$ cells using the miRNeasy Mini kit (Qiagen) according to the manufacturer's instructions. cDNA was synthesized from $500 \mathrm{ng}$ of the total RNA using the RT ${ }^{2}$ First Strand Kit (Qiagen), which includes the additional removal of genomic DNA from the RNA sample and a specific control of reverse transcription. The quality of isolated RNA was also evaluated using $\mathrm{RT}^{2}$ RNA QC PCR Arrays (Qiagen) according to the manufacturer's instructions. This test includes various measures allowing to control the presence of reverse transcription and PCR inhibitors, contamination with genomic DNA, and contamination with DNA during the procedure.

After all control tests, the samples were analysed using the $\mathrm{RT}^{2}$ Profiler PCR Array. Altogether, 84 different genes were simultaneously amplified in the sample. A melting curve analysis was performed to verify that the product consisted of a single amplicon. PCR arrays were performed 
in 384-well plates on a LightCycler 480 instrument (Roche Applied Science). Briefly, the reaction mix was prepared from 2x SABiosciences $\mathrm{RT}^{2}$ qPCR Master Mix and $102 \mu \mathrm{L}$ of sample cDNA. $10 \mu \mathrm{L}$ of this mixture was added into each well of the PCR Array. The data were analysed via Roche LightCycler 480 software and the $C_{t}$ values were extracted for each gene. The thresholds and baselines were set according to the manufacturer's instructions (SABiosciences, Qiagen). The data were analysed using software supplied by Qiagen (http://www.sabiosciences.com/pcr/arrayanalysis.php). The fold change in gene expression (compared to positive control BM-MSCs) was calculated using the $\Delta \Delta C_{t}$ method. A more than threefold change in gene expression (compared to positive control BM-MSCs) was considered as the up- or downregulation of a specific gene expression.

2.4. Alkaline Phosphatase Activity Staining. An alkaline phosphatase detection kit (Millipore) was used for staining of alkaline phosphatase (AP) activity. Briefly, the AFCs were fixed in $4 \%$ paraformaldehyde (PFA) for $1 \mathrm{~min}$, washed with PBS, and incubated for $15 \mathrm{~min}$ in a working solution of reagents, which consisted of Fast Red Violet, Naphthol ASBI phosphate solution and water in a 2:1:1 ratio. The culture was observed under an inverted microscope (Hoffman illumination) to confirm AP activity. The cells or cell clusters expressing AP activity were stained from pink to violet.

2.5. Differentiation of AFCs into Osteogenic-, Adipogenic-, and Pancreatic-Like Cells. Osteogenic differentiation was induced using the well-known osteogenic differentiation medium [17]. It consisted of DMEM low glucose, Lglutamine, FBS, dexamethasone (Sigma), L-ascorbic acid 2phosphate (Sigma), $\beta$-Glycerophosphate (Sigma), and penicillin/streptomycin. To confirm successful differentiation, the cell culture was stained using the von Kossa protocol after 12-14 days of differentiation. The cells were fixed in a $4 \%$ PFA, incubated in $2 \%$ silver nitrate in the dark for 10 minutes, washed with distilled water, and exposed to UV-light for 25 minutes. After washing, the cells were observed under an inverted microscope to detect the calcium deposits, which were stained black.

To induce adipogenic differentiation, an induction medium was used as previously described [16]. The cells were cultured in a medium consisting of hESC medium (DMEM/F12, 20\% KnockOut Serum Replacement (Gibco), $1 \mathrm{mM}$ L-glutamine (PAA), $1 \%$ nonessential amino acids (PAA), $0.1 \mathrm{mM}$ 2-mercaptoethanol (Invitrogen), $13 \mathrm{mM}$ HEPES, $8 \mathrm{ng} / \mathrm{mL}$ human basic fibroblast growth factor (bFGF, Sigma-Aldrich), and $1 \%$ penicillin/streptomycin) and $20 \%$ FF. The differentiation medium was changed every 3-4 days. After 2 weeks, the cells were fixed in a $4 \%$ PFA for 20 minutes and incubated for 10 minutes in an Oil Red $\mathrm{O}$ working solution. After thorough washes, the cells were observed under an inverted microscope for presence of lipid droplets, which were stained red.

To induce pancreatic differentiation, the cells were cultured according to the protocol of Chandra et al. [18] which was slightly modified. Briefly, the cells were cultured for two days in SFM medium (serum free medium; DMEM/F12, 1\% ITS, $1 \% \mathrm{BSA}$ ) supplemented with $4 \mathrm{nM}$ activin $\mathrm{A}, 50 \mu \mathrm{M} 2-$ mercaptoethanol, and $2 \mathrm{ng} / \mathrm{mL}$ bFGF. On the third day, the medium was changed to SFM supplemented with $0.3 \mathrm{mM}$ taurine and on the fifth day to SFM supplemented with $3 \mathrm{mM}$ taurine, $1 \mathrm{mM}$ nicotinamide, and $1 \%$ nonessential amino acids. After 10-14 days, the cells were analysed by using dithizone staining. Briefly, the stock solution of dithizone was prepared by dissolving $10 \mathrm{mg}$ of dithizone in $1 \mathrm{~mL}$ of dimethyl sulfoxide (DMSO). Then, $10 \mu \mathrm{L}$ of stock solution was added to $1 \mathrm{~mL}$ of DMEM/F12 and filtered through a $0.4 \mu \mathrm{m}$ filter, and cells were incubated in this working solution for $15 \mathrm{~min}$ at $37^{\circ} \mathrm{C}$. After incubation, the cells were washed 4 times with PBS and observed under an inverted microscope. Positively stained cells were coloured red.

\section{Results}

3.1. Expression of MSCs-Related Genes in AFCs and Fibroblasts in Comparison to BM-MSCs. Expression of 57 genes was detected in AFCs when compared with BM-MSCs (positive control) (Table 1). Sixteen genes were upregulated in AFCs, among which MSC-associated genes IL10 and CD45 were two of the most upregulated genes with fold change of almost 1100 and 900, respectively. Fold change between 30 and 40 was detected for MSC-specific or associated genes CD49f, TNF, $I L 1 B$, and adipogenesis- and osteogenesis-related RUNX2. Two highly upregulated genes were also MSC-specific or associated genes CD106 and VWF with fold change of around 20 and 15, respectively. All other genes (OCT4, CD146, CD54, VEGF, HDAC1, MITF, PPARG, and PCAF) showed fold change between 3 and 10 (Figure 1(a)). Twenty genes were downregulated in AFCs when compared with BM-MSCs, among which MSC-specific or associated genes COL1A1, $M M P 2$, and $P D G F R B$ were the most downregulated genes with fold changes -266 (COL1A1), -225 (MMP2), and -119 (PDGFRB). Highly downregulated genes were also FGF2, CD73, CD90, NUDT6, NES, and CD105, with fold changes between -33 and -12 , respectively. All other genes (GDF5, CASP3, CD13, CD340, KDR, BDNF, IL6, BMP6, SMURF2, $B M P 4$, and $J A G 1$ ) showed fold change between -3 and -10 (Figure 1(b)). There were 27 genes which were not detected in AFCs; about one-third of them was stemness or MSCsspecific genes; one-third was genes associated with MSCs, and one-third was osteogenesis- or chondrogenesis-related genes. All these data showed that cultured AFCs expressed several genes specific or associated with MSCs, but the expression pattern was different than in BM-MSCs. Similar to BM-MSCs, AFCs did not express the key genes related to stemness or pluripotency (SOX2, REX1, TERT, WNT3A, and INS) or expressed them at very low level (OCT4 and LIF).

In AFCs, there was a higher number of upregulated genes than in HDFs (negative control) in comparison with BMMSCs. In AFCs, other set of MSC-specific or associated genes (CD49f, CD106, CD146, CD45, CD54, IL10, IL1B, $T N F, V E G F$, and $V W F$ ) were prominently upregulated than in HDFs (CD90 and KITLG). In HDFs, the expression of lower number of genes was detected than in AFCs. 
TABLE 1: Expression levels of 84 genes in adult human dermal fibroblasts and aspirated follicular cells in comparison with bone marrow-derived mesenchymal stem cells, respectively.

\begin{tabular}{|c|c|c|c|}
\hline \multirow{2}{*}{ Gene name } & \multicolumn{2}{|c|}{ Expression level } & \multirow{2}{*}{$\begin{array}{l}\text { REFSEQ } \\
\text { (mRNA) }\end{array}$} \\
\hline & Fibroblasts & $\begin{array}{l}\text { Aspirated } \\
\text { follicular cells }\end{array}$ & \\
\hline$A B C B 1$ & - & - & NM_000927 \\
\hline ANXA5 & 2.17 & -1.50 & NM_001154 \\
\hline$B D N F$ & -16.45 & -8.40 & NM_001143805 \\
\hline$B G L A P$ & -1.79 & -1.49 & NM_199173 \\
\hline$B M P 2$ & - & - & NM_001200 \\
\hline$B M P 4$ & 2.01 & -8.06 & NM_001202 \\
\hline BMP6 & -5.82 & -9.32 & NM_001718 \\
\hline$B M P 7$ & - & - & NM_001719 \\
\hline CASP3 & -1.44 & -3.36 & NM_004346 \\
\hline CD105 & -2.64 & -12.04 & NM_000118 \\
\hline CD106 & - & 19.56 & NM_001078 \\
\hline CD11c & - & - & NM_000887 \\
\hline CD13 & 1.61 & -4.93 & NM_001150 \\
\hline CD133 & - & - & NM_001139319 \\
\hline CD146 & - & 5.98 & NM_006500 \\
\hline CD15 & - & - & NM_002033 \\
\hline CD166 & -7.06 & 1.36 & NM_001243280 \\
\hline$C D 271$ & - & - & NM_002507 \\
\hline CD29 & 1.77 & 1.17 & NM_002211 \\
\hline CD340 & -1.65 & -5.17 & NM_001005862 \\
\hline CD349 & - & - & NM_003508 \\
\hline CD44 & 1.51 & -2.55 & NM_000610 \\
\hline CD45 & - & 891.44 & NM_001267798 \\
\hline$C D 49 f$ & 1.01 & 37.53 & NM_000210 \\
\hline CD51 & -1.55 & -1.71 & NM_001144999 \\
\hline CD54 & 1.15 & 4.79 & NM_000201 \\
\hline$C D 73$ & -1.03 & -23.26 & NM_001204813 \\
\hline CD90 & 19.70 & -32.90 & NM_006288 \\
\hline COL1A1 & -9.06 & -266.87 & NM_000088 \\
\hline CSF2 & - & - & NM_000758 \\
\hline CSF3 & - & - & NM_000759 \\
\hline CTNNB1 & 2.35 & 1.84 & NM_001098209 \\
\hline$E G F$ & - & - & NM_001178130 \\
\hline FGF10 & - & - & NM_004465 \\
\hline$F G F 2$ & -1.45 & -21.71 & NM_002006 \\
\hline FUT1 & - & - & NM_000148 \\
\hline GDF15 & -19.70 & -1.89 & NM_004864 \\
\hline GDF5 & 11.88 & -5.28 & NM_000557 \\
\hline GDF6 & - & - & NM_001001557 \\
\hline GDF7 & - & - & NM_182828 \\
\hline GTF3A & -2.00 & 1.82 & NM_002097 \\
\hline HAT1 & 1.23 & 1.75 & NM_001033085 \\
\hline$H D A C 1$ & 3.48 & 3.58 & NM_004964 \\
\hline$H G F$ & - & - & NM_000601 \\
\hline HNF1A & - & - & NM_000545 \\
\hline IFNG & - & - & NM_000619 \\
\hline
\end{tabular}

TABLE 1: Continued.

\begin{tabular}{|c|c|c|c|}
\hline \multirow{2}{*}{ Gene name } & \multicolumn{2}{|c|}{ Expression level } & \multirow{2}{*}{$\begin{array}{l}\text { REFSEQ } \\
\text { (mRNA) }\end{array}$} \\
\hline & Fibroblasts & $\begin{array}{l}\text { Aspirated } \\
\text { follicular cells }\end{array}$ & \\
\hline IGF1 & - & - & NM_000618 \\
\hline IL10 & - & 1074.91 & NM_000572 \\
\hline$I L 1 B$ & -41.07 & 33.83 & NM_000576 \\
\hline IL6 & -27.67 & -4.76 & NM_000600 \\
\hline INS & - & - & NM_000207 \\
\hline$J A G 1$ & -1.44 & -9.99 & NM_000214 \\
\hline$K D R$ & -12.04 & -3.25 & NM_002253 \\
\hline KITLG & 3.97 & -1.87 & NM_000899 \\
\hline LIF & - & -1.20 & NM_001257135 \\
\hline MITF & 1.63 & 4.82 & NM_000248 \\
\hline MMP2 & 1.97 & -225.97 & NM_001127891 \\
\hline NES & -48.50 & -16.00 & NM_006617 \\
\hline NOTCH1 & - & - & NM_017617 \\
\hline NUDT6 & -1.49 & -11.88 & NM_007083 \\
\hline OCT4 & 2.27 & 4.63 & NM_001173531 \\
\hline PCAF & 4.23 & 6.11 & NM_003884 \\
\hline PDGFRB & 2.30 & -119.43 & NM_002609 \\
\hline PIGS & -1.49 & 2.48 & NM_033198 \\
\hline PPARG & 1.80 & 7.67 & NM_005037 \\
\hline PTK2 & 1.04 & -1.14 & NM_001199649 \\
\hline$R E X 1$ & - & - & NM_174900 \\
\hline RHOA & -1.15 & -1.52 & NM_001664 \\
\hline RUNX2 & 1.68 & 30.70 & NM_001015051 \\
\hline SLC17A5 & 2.25 & -1.51 & NM_012434 \\
\hline SMAD4 & 4.11 & 2.06 & NM_005359 \\
\hline SMURF1 & 1.14 & 1.23 & NM_001199847 \\
\hline SMURF2 & -2.51 & -8.75 & NM_022739 \\
\hline SOX2 & - & - & NM_003106 \\
\hline SOX9 & - & 1.72 & NM_000346 \\
\hline TBX2 & - & - & NM_005994 \\
\hline TERT & - & - & NM_001193376 \\
\hline TGFB1 & -1.54 & 2.03 & NM_000660 \\
\hline TGFB3 & 1.01 & 1.28 & NM_003239 \\
\hline$T N F$ & - & 35.75 & NM_000594 \\
\hline VEGF & -4.44 & 3.10 & NM_001025366 \\
\hline$V I M$ & -1.53 & -1.87 & NM_003380 \\
\hline$V W F$ & - & 14.42 & NM_000552 \\
\hline WNT3A & - & - & NM_033131 \\
\hline
\end{tabular}

-: expression of the gene was not detected.

The expression of 50 genes was detected in HDFs when compared with BM-MSCs (Table 1). A lower number -6 genes were upregulated in HDFs, among which MSC-specific $C D 90$ was the most upregulated gene with fold change of around 20. All other upregulated genes $(K I T L G$-associated with MSCs, $H D A C 1$ - osteogenesis, $P C A F$-chondrogenesis, and SMAD4-tenogenesis) had fold change of around 4, with exception of chondrogenesis-related GDF5, which had fold change of around 10 (Figure 2(a)). Ten genes were downregulated in HDFs when compared with BM-MSCs, 


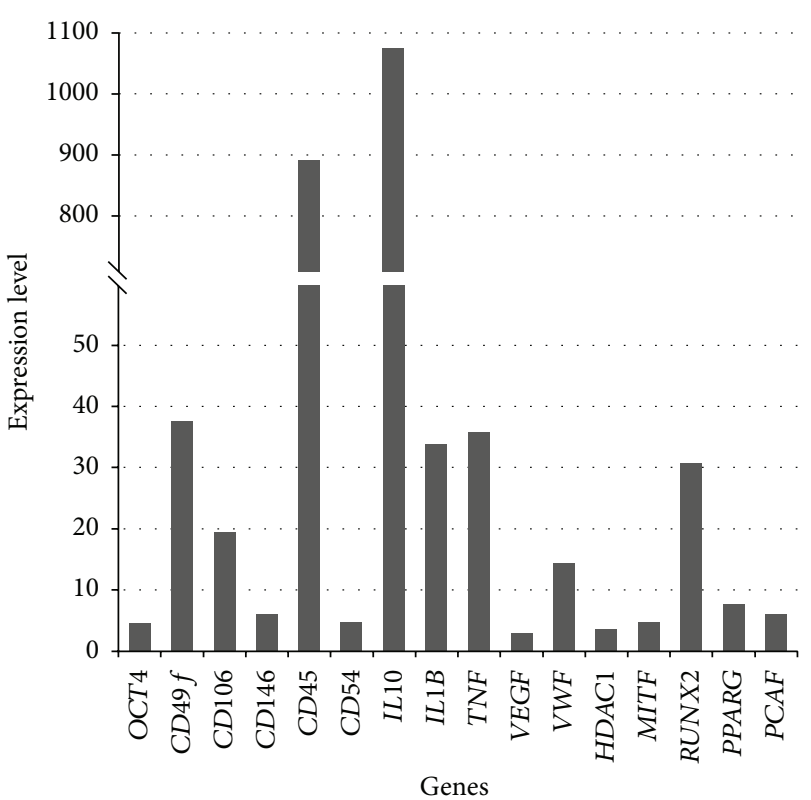

(a)

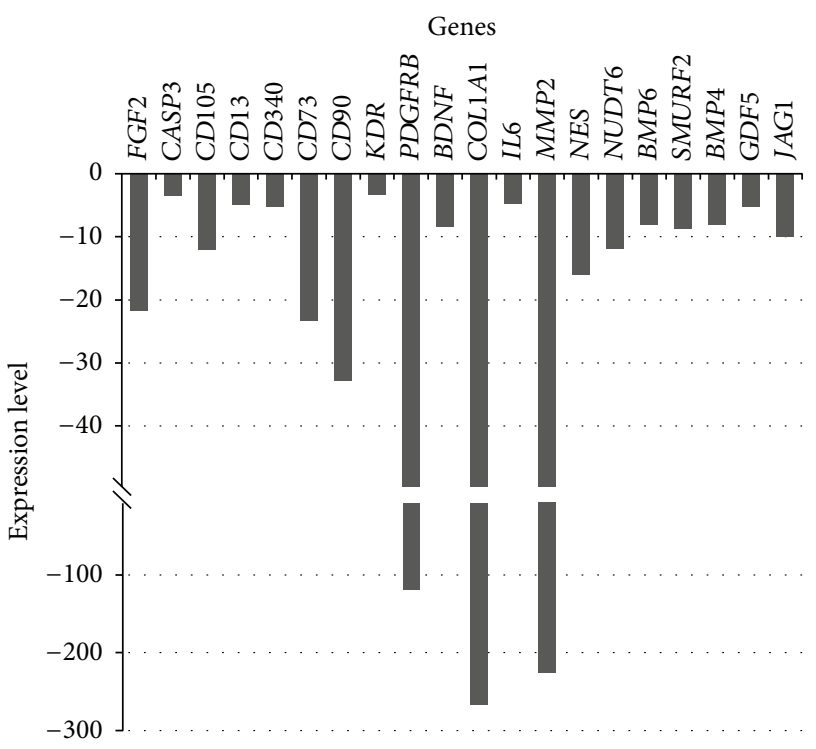

(b)

FIGURE 1: Expression levels of upregulated (a) and downregulated (b) genes in aspirated follicular cells obtained from follicular aspirates when compared with bone marrow-derived mesenchymal stem cells (positive control).

among which MSC-specific NES, IL1B, and IL6 were the most downregulated genes with fold change of around -30 (IL6), of around -40 (IL1B), and of around -50 (NES). Fold change between -10 and -20 was detected for GDF15, $B D N F$, and KDR genes. All other genes (CD166, COL1A1, $V E G F$, and BMP6) showed fold change between -10 and -3 (Figure 2(b)).

3.2. Culturing of AFCs and Differentiation in Other Cell Types. Immediately after transferring enriched AFCs from follicular

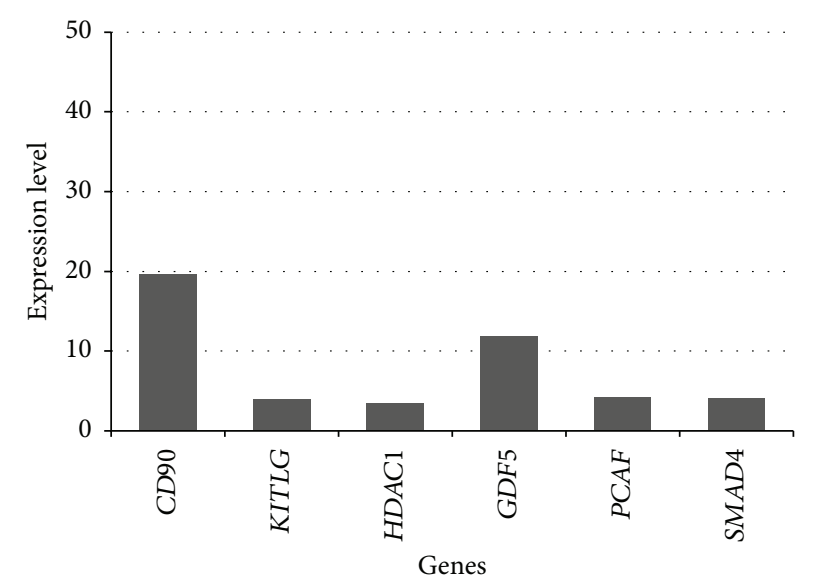

(a)

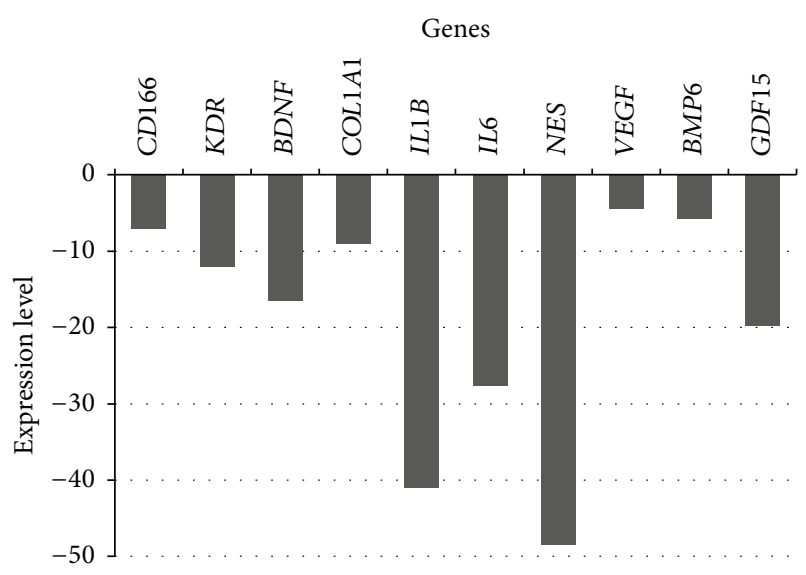

(b)

FIGURE 2: Expression levels of upregulated (a) and downregulated (b) genes in adult human dermal fibroblasts (negative control) when compared with bone marrow-derived mesenchymal stem cells (positive control).

aspirates into culture dish, we observed clusters of AFCs with approximately $100 \mu \mathrm{m}$ in diameter and also single AFCs with numerous surrounding red blood cells (Figure 3(a)) which were not removed with hypoosmotic protocol. After AFCs were attached to a culture dish surface, red blood cells were removed upon washing with PBS and first change of the culture medium (on the second day). AFCs exhibited fibroblast-like phenotype (Figure 3(b)), although epitheliallike AFCs were also observed in minority. After 48 hours, AFCs also started migrating from packed clusters. We were able to maintain AFCs alive for 2 months; however, viability (attachment to the surface and cell proliferation) of AFCs decreased with every passage, but it was unique case with every patient.

Cultured AFCs were highly positive for AP, and around 60\% AFCs showed strongly pink-violet staining (Figure 3(c)) throughout the culturing. When AFCs were exposed to media for osteogenic differentiation, cell morphology was slightly changed; they shrunk, and around $10 \%$ of AFCs 


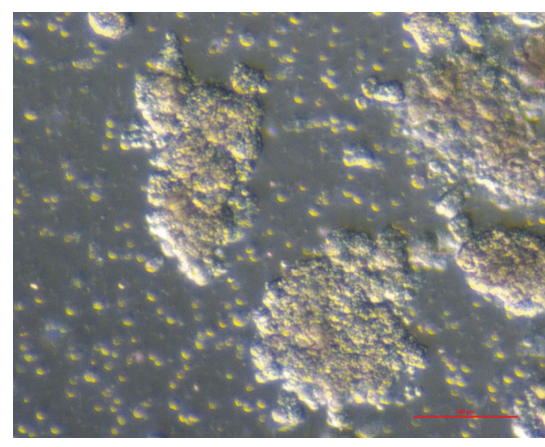

(a)

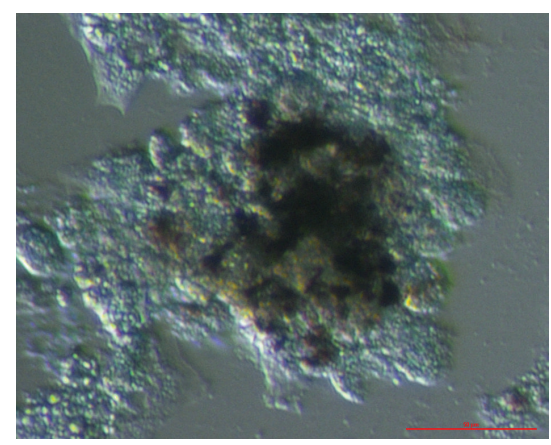

(d)

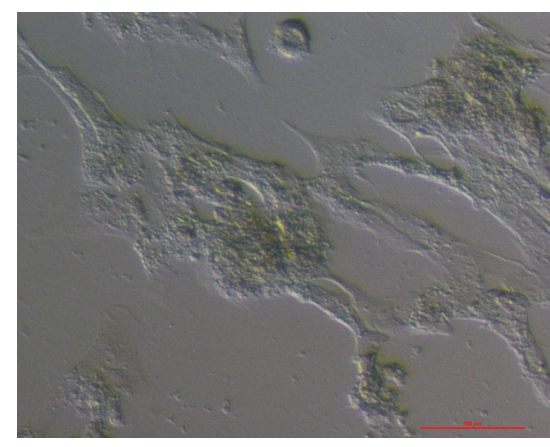

(b)

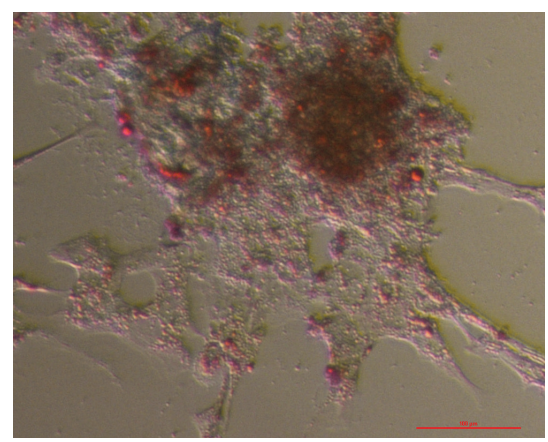

(e)

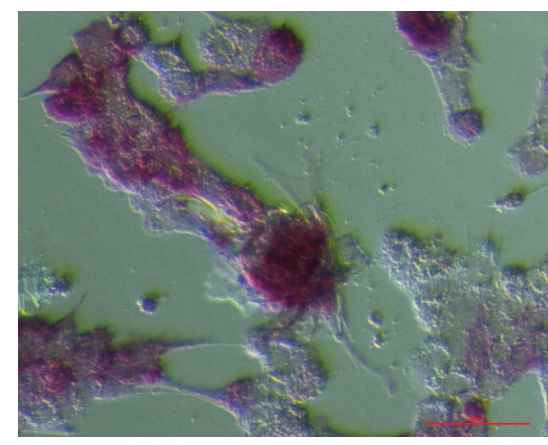

(c)

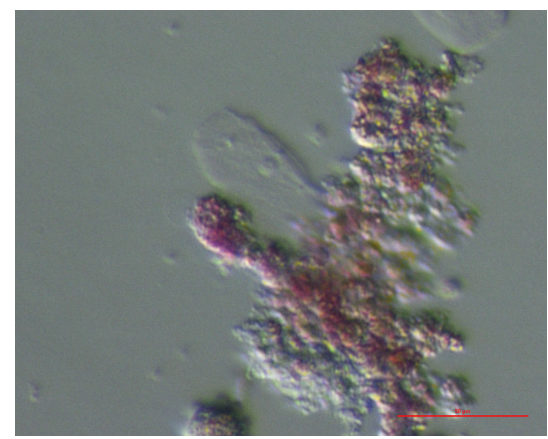

(f)

FIGURE 3: Epithelial-like phenotype of aspirated follicular cells (AFCs) in culture dish immediately after enrichment with hypoosmotic method (a). Fibroblast-like phenotype of AFCs in culture dish 48 hours after isolation (b). AFCs positive for alkaline phosphatase activity (pink-violet) (c). Differentiation of AFCs into osteogenic-like cells, von Kossa-positive staining (d). Differentiation of AFCs into adipogeniclike cells, accumulation of lipid droplets (dark red) (e). Differentiation of AFCs into pancreatic-like cells, dithizone-positive (bright red-pink) cell cluster (f). Scale bar: $100 \mu \mathrm{m}$ (a, b, c, e) and $50 \mu \mathrm{m}$ (d, f).

stained positively for mineralization (Figure 3(d)). Additionally, when AFCs were exposed to media for adipogenic differentiation, accumulation of lipid droplets was observed throughout the cell culture (Figure 3(e)). AFCs were also exposed to media for pancreatic differentiation. Cell morphology was changed forming clusters of islet-like structures and around $5 \%$ of cells positively stained on dithizone (Figure 3(f)).

\section{Discussion}

In this study, AFCs obtained from follicular aspirates of infertile women included into the in vitro fertilization programme were successfully cultured and their stemness was confirmed. The gene expression profile and in vitro differentiation of cultured AFCs into other cell types confirmed the relation of AFCs to MSCs, but their stemness was specific and it differed from BM-MSCs and fibroblasts.

The in vitro culturing and research of molecular and cellular characteristics of AFCs and their subpopulations such as GCs or TCs are still difficult since there is no ultimate protocol for their purification from follicular aspirates. Subpopulations of AFCs can be isolated by flow cytometry based on the expression of specific cell marker, for example, follicle-stimulating hormone receptor (FSHR) for isolation of GCs [8]; however, this approach can lead to a loss of less differentiated/progenitor GCs which do not express FSHR yet. In this study, we used the hypoosmotic purification protocol described by Lobb and Younglai [15] to enrich AFCs because it is quite simple and can be quickly done, removes most of red blood cells from the sample, and yields more AFCs in comparison with multistep protocols. The follicular aspirates also contain a proportion of white blood cells which represent approximately $15 \%$ of all cells [19] and are unavoidable contaminant. On the other hand, these "contaminating" cells could play an important role in maintaining a more physiological ovarian stem cell niche [20].

In this study, we successfully established a long-term culture of AFCs. In previous studies, the apoptosis represented a major problem in AFCs culturing and research. However, we found for the first time that the addition of follicular fluid serum to the culture medium enables a long-term survival of AFCs in vitro. Because the potential use of AFCs is related to their culture and proliferation in vitro, we were interested in gene expression analysis of cultured AFCs more than freshly isolated. However, in vitro culturing can significantly affect the gene expression of cells as previously shown in human stromal cells [21]. Even more, for some AFCs like GCs, it has been demonstrated that they can undergo dedifferentiation in vitro and downregulation of GCs-specific genes may occur after 96 hours of culturing [9]. 
Our data showed that all three groups of analyzed cells expressed a proportion of MSC-specific or associated genes thus reflected the same-mesodermal-origin of cells. In spite of that, the gene expression profile of AFCs, BM-MSCs, and $\mathrm{HDFs}$ was different and indicated three distinct groups of cells. There were eight genes which were expressed in both the AFCs and BM-MSCs, but were not expressed in HDFs; these genes were related to stemness $(L I F)$ and were MSCsspecific (CD106 and CD146), associated with MSCs (IL10, CD45, TNF, and VWF) or chondrogenesis related (SOX9). In AFCs, several MSCs-specific or associated genes were upregulated. The AFCs were not only characterized by a very high expression of genes IL10 and CD45 that may reflect their association with MSCs, but also to a lower extent the contamination with blood cells. The gene ILIO is known to be related to immunoregulation (inflammation), while the gene CD45 encodes the protein belonging to the tyrosine phosphatase (PTP) family; the PTPs are known to be signaling molecules that regulate a variety of cellular processes including cell growth, differentiation, mitosis, and oncogenic transformation according to the GeneCard database.

The results of this study show that AFCs expressed several genes typical for somatic ovarian cells, especially GCs. In addition, the morphology of AFCs clusters resembled the GCs; therefore, it is not excluded that GCs represented majority of cells in our cell cultures. The expression of gene $V E G F$, vascular endothelial growth factor, was previously demonstrated in GCs and was shown to be very important factor in controlling angiogenesis during development of corpus luteum [22]. In addition, CD146, melanoma cell adhesion molecule, was shown to be expressed on human luteinizing GCs [23]. CD49f, also known as integrin alpha6 , has been demonstrated to be expressed on the surface of human GCs and represents a differentiation marker of GCs [24]; it was found to be more distinctive for GCs from the inner layers of follicle [25]. The gene PPAPRG, peroxisome proliferator-activated receptor gamma, encodes a nuclear hormone receptor which is related to steroid hormone action [26]. The activity of GCs is strongly influenced by folliclestimulating hormone and luteinizing hormone [27]. The gene $H D A C l$, histone deacetylase 1 , is one of the important regulators of human luteinizing hormone receptor gene transcription [28]. In AFCs, also some genes related to osteogenesis and adipogenesis were upregulated; MITF has been connected with osteogenesis [29], along with RUNX2 [30]. In addition, PCAF was recently shown to acetylate RUNX2 which leads to transcriptional activity and thus promotes osteoblast differentiation [31]. In AFCs, there was a higher number of upregulated genes related to MSCs than in HDFs in comparison with BM-MSCs and other set of MSC-specific or associated genes was prominently upregulated than in HDFs. In addition, the genes upregulated in HDFs were more related to cell differentiation (osteogenesis, chondrogenesis, tenogenesis) than to stemness thus indicating that HDFs were more differentiated cells than cultured AFCs.

The AFCs were not pluripotent stem cells, because they did not express genes related to pluripotency such as REX1, SOX2, TERT, and WNT3A. In spite of that, they expressed two pluripotency-related genes: OCT4 and LIF to a lower extent. The expression of OCT4 in AFCs probably reflects the presence of GCs as previously confirmed by other studies [8, $32,33]$. However, OCT4 was also expressed in both BM-MSCs and HDFs to the same extent; therefore, the nonspecificity of primer for OCT4A, related to pluripotent stem cells [34], is not excluded. It needs to be exposed that the LIF gene, an important marker of stemness [35], was detected to the same extent in AFCs and BM-MSCs, but was not detected in HDF; this might reflect a lower stemness of HDFs.

A subpopulation of AFCs expressed a degree of plasticity, because we were able to successfully differentiate them into osteogenic, adipocyte and pancreatic-like cells. AFCs seem to be especially in favour of osteogenesis thus reflecting the presence of GCs, as evidenced by other studies [9, 36]. In our experiments, AFCs strongly expressed the gene RUNX2 which is involved in osteogenesis [37] and GCs luteinization [38], differentiated into osteogenic-like cells confirmed by Von Kossa staining and stained positively for alkaline phosphatase activity which is considered as an early marker of osteogenesis [39]. Moreover, AFCs were successfully differentiated into adipose and pancreatic-like cells in this study. To our knowledge differentiation of AFCs into adipocyte and pancreatic-like cells has not been reported until now; therefore, our work additionally supports the idea about the stemness and plasticity of human AFCs.

\section{Conclusion}

In conclusion, the results of our study showed that AFCs enriched from follicular aspirates of infertile women using hypoosmotic protocol and cultured in vitro expressed 57 from 84 analyzed genes related to stemness, MSCs, and cell differentiation. Numerous upregulated genes were specific for MSCs or were associated with them. The expression of these genes confirmed the stemness of AFCs in our cultures; however, the gene expression profile differed from that of BMMSCs. The gene expression profile of AFCs also differed from that of HDFs which were found to be more differentiated cells. In AFCs, also several expressed genes were related to the ovary and its function. The AFCs expressed a degree of plasticity and were successfully differentiated into other types of cells which are otherwise not present in the ovary.

\section{Conflict of Interests}

The authors declare that there is no conflict of interests.

\section{Acknowledgments}

The authors would like to thank all the patients whose follicular aspirates were used for this research.

\section{References}

[1] M. Hamel, I. Dufort, C. Robert et al., "Identification of differentially expressed markers in human follicular cells associated with competent oocytes," Human Reproduction, vol. 23, no. 5, pp. 1118-1127, 2008. 
[2] G. F. Erickson, "Follicle growth and development," in Gynecology and Obstetrics CD-ROM, J. J. Sciarra, Ed., chapter 12, Lippincott Williams and Wilkins, Philadelphia, Pa, USA, 2004.

[3] M. W. Beckmann, D. Polacek, L. Seung, and J. R. Schreiber, "Human ovarian granulosa cell culture: determination of blood cell contamination and evaluation of possible culture purification steps," Fertility and Sterility, vol. 56, no. 5, pp. 881-887, 1991.

[4] J. A. Loukides, R. A. Loy, R. Edwards, J. Honig, I. Visintin, and M. L. Polan, "Human follicular fluids contain tissue macrophages," Journal of Clinical Endocrinology and Metabolism, vol. 71, no. 5, pp. 1363-1367, 1990.

[5] K. Kossowska-Tomaszczuk and C. De Geyter, "Cells with stem cell characteristics in somatic compartments of the ovary," BioMed Research International, vol. 2013, Article ID 310859, 8 pages, 2013.

[6] E. Dzafic, M. Stimpfel, and I. Virant-Klun, "Plasticity of granulosa cells: on the crossroad of stemness and transdifferentiation potential," Journal of Assisted Reproduction and Genetics, vol. 30, no. 10, pp. 1255-1261, 2013.

[7] A. Gougeon, "Dynamics of follicular growth in the human: a model from preliminary results," Human Reproduction, vol. 1, no. 2, pp. 81-87, 1986.

[8] K. Kossowska-Tomaszczuk, C. De Geyter, M. De Geyter et al., "The multipotency of luteinizing granulosa cells collected from mature ovarian follicles," Stem Cells, vol. 27, no. 1, pp. 210-219, 2009.

[9] Y. Oki, H. Ono, T. Motohashi, N. Sugiura, H. Nobusue, and K. Kano, "Dedifferentiated follicular granulosa cells derived from pig ovary can transdifferentiate into osteoblasts," Biochemical Journal, vol. 447, no. 2, pp. 239-248, 2012.

[10] J. L. Pitman, A. S. McNeilly, J. R. McNeilly et al., "The fate of granulosa cells following premature oocyte loss and the development of ovarian cancers," The International Journal of Developmental Biology, vol. 56, no. 10-12, pp. 949-958, 2012.

[11] A. Honda, M. Hirose, K. Hara et al., "Isolation, characterization, and in vitro and in vivo differentiation of putative thecal stem cells," Proceedings of the National Academy of Sciences of the United States of America, vol. 104, no. 30, pp. 12389-12394, 2007.

[12] Y. Takehara, A. Yabuuchi, K. Ezoe et al., "The restorative effects of adipose-derived mesenchymal stem cells on damaged ovarian function," Laboratory Investigation, vol. 93, no. 2, pp. 181-193, 2013.

[13] X. Fu, Y. He, C. Xie, and W. Liu, "Bone marrow mesenchymal stem cell transplantation improves ovarian function and structure in rats with chemotherapy-induced ovarian damage," Cytotherapy, vol. 10, no. 4, pp. 353-363, 2008.

[14] K. Knez, T. Tomazevic, B. Zorn, E. Vrtacnik-Bokal, and I. Virant-Klun, "Intracytoplasmic morphologically selected sperm injection improves development and quality of preimplantation embryos in teratozoospermia patients," Reproductive Biomedicine Online, vol. 25, no. 2, pp. 168-179, 2012.

[15] D. K. Lobb and E. V. Younglai, "A simplified method for preparing IVF granulosa cells for culture," Journal of Assisted Reproduction and Genetics, vol. 23, no. 2, pp. 93-95, 2006.

[16] M. Stimpfel, T. Skutella, M. Kubista, E. Malicev, S. Conrad, and I. Virant-Klun, "Potential stemness of frozen-thawed testicular biopsies without sperm in infertile men included into the in vitro fertilization programme," Journal of Biomedicine and Biotechnology, vol. 2012, Article ID 291038, 15 pages, 2012.

[17] P. A. Zuk, M. Zhu, H. Mizuno et al., "Multilineage cells from human adipose tissue: Implications for cell-based therapies," Tissue Engineering, vol. 7, no. 2, pp. 211-228, 2001.
[18] V. Chandra, S. Phadnis, P. D. Nair, and R. R. Bhonde, "Generation of pancreatic hormone-expressing islet-like cell aggregates from murine adipose tissue-derived stem cells," Stem Cells, vol. 27, no. 8, pp. 1941-1953, 2009.

[19] H. Ferrero, F. Delgado-Rosas, C. M. Garcia-Pascual et al., "Efficiency and purity provided by the existing methods for the isolation of luteinized granulosa cells: a comparative study," Human Reproduction, vol. 27, no. 6, pp. 1781-1789, 2012.

[20] A. Bukovsky, "Ovarian stem cell niche and follicular renewal in mammals," Anatomical Record, vol. 294, no. 8, pp. 1284-1306, 2011.

[21] A. C. Boquest, A. Shahdadfar, K. Frønsdal et al., "Isolation and transcription profiling of purified uncultured human stromal stem cells: alteration of gene expression after in vitro cell culture," Molecular Biology of the Cell, vol. 16, no. 3, pp. 1131-1141, 2005.

[22] Y. Qiu, M. Seager, A. Osman et al., “Ovarian VEGF165b expression regulates follicular development, corpus luteum function and fertility," Reproduction, vol. 143, no. 4, pp. 501-511, 2012.

[23] S. Yoshioka, H. Fujiwara, T. Higuchi, S. Yamada, M. Maeda, and S. Fujii, "Melanoma cell adhesion molecule (MCAM/CD146) is expressed on human luteinizing granulosa cells: enhancement of its expression by hCG, interleukin-1 and tumour necrosis factor- $\alpha$," Molecular Human Reproduction, vol. 9, no. 5-6, pp. 311-319, 2003.

[24] T. Honda, H. Fujiwara, M. Ueda, M. Maeda, and T. Mori, "Integrin $\alpha 6$ is a differentiation antigen of human granulosa cells," Journal of Clinical Endocrinology and Metabolism, vol. 80, no. 10, pp. 2899-2905, 1995.

[25] H. Fujiwara, M. Maeda, T. Honda et al., "Granulosa cells express integrin $\alpha 6$ : possible involvement of integrin $\alpha 6$ in folliculogenesis," Hormone Research, vol. 46, no. 1, pp. 24-30, 1996.

[26] M. P. Kowalewski, M. T. Dyson, P. R. Manna, and D. M. Stocco, "Involvement of peroxisome proliferator-activated receptor in gonadal steroidogenesis and steroidogenic acute regulatory protein expression," Reproduction, Fertility and Development, vol. 21, no. 7, pp. 909-922, 2009.

[27] R. Canipari, V. Cellini, and S. Cecconi, "The ovary feels fine when paracrine and autocrine networks cooperate with gonadotropins in the regulation of folliculogenesis," Current Pharmaceutical Design, vol. 18, no. 3, pp. 245-255, 2012.

[28] Y. Zhang and M. L. Dufau, "Silencing of transcription of the human luteinizing hormone receptor gene by histone deacetylase-mSin3A complex," Journal of Biological Chemistry, vol. 277, no. 36, pp. 33431-33438, 2002.

[29] S. M. Sharma, S. Sif, M. C. Ostrowski, and U. Sankar, "Defective co-activator recruitment in osteoclasts from microphthalmiaoak ridge mutant mice," Journal of Cellular Physiology, vol. 220, no. 1, pp. 230-237, 2009.

[30] R. T. Franceschi and G. Xiao, "Regulation of the osteoblastspecific transcription factor, Runx2: responsiveness to multiple signal transduction pathways," Journal of Cellular Biochemistry, vol. 88, no. 3, pp. 446-454, 2003.

[31] C. Y. Wang, S. F. Yang, Z. Wang et al., "PCAF acetylates Runx2 and promotes osteoblast differentiation," Journal of Bone and Mineral Metabolism, vol. 31, no. 4, pp. 381-389, 2013.

[32] M. Varras, T. Griva, V. Kalles, C. Akrivis, and N. Paparisteidis, "Markers of stem cells in human ovarian granulosa cells: is there a clinical significance in ART?" Journal of Ovarian Research, vol. 5, no. 1, article 36, 2012. 
[33] H. Patel, D. Bhartiya, S. Parte, P. Gunjal, S. Vedulkar, and M. Bhatt, "Follicle stimulating hormone modulates ovarian stem cells through alternetly spliced receptor variant FSH-R3," Journal of Ovarian Research, vol. 6, no. 1, article 52, 2013.

[34] X. Wang and J. Dai, "Concise review: isoforms of OCT4 contribute to the confusing diversity in stem cell biology," Stem Cells, vol. 28, no. 5, pp. 885-893, 2010.

[35] H. Hirai, P. Karian, and N. Kikyo, "Regulation of embryonic stem cell self-renewal and pluripotency by leukaemia inhibitory factor," Biochemical Journal, vol. 438, no. 1, pp. 11-23, 2011.

[36] M. Mattioli, A. Gloria, M. Turriani et al., "Osteo-regenerative potential of ovarian granulosa cells: an in vitro and in vivo study," Theriogenology, vol. 77, no. 7, pp. 1425-1437, 2012.

[37] T. Komori, "Regulation of osteoblast differentiation by runx2," Advances in Experimental Medicine and Biology, vol. 658, pp. 43-49, 2010.

[38] E. S. Park, J. Park, R. T. Franceschi, and M. Jo, “The role for runt related transcription factor 2 (RUNX2) as a transcriptional repressor in luteinizing granulosa cells," Molecular and Cellular Endocrinology, vol. 362, no. 1-2, pp. 165-175, 2012.

[39] C. D. Hoemann, H. El-Gabalawy, and M. D. McKee, "In vitro osteogenesis assays: influence of the primary cell source on alkaline phosphatase activity and mineralization," Pathologie Biologie, vol. 57, no. 4, pp. 318-323, 2009. 


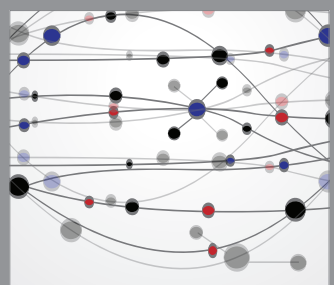

The Scientific World Journal
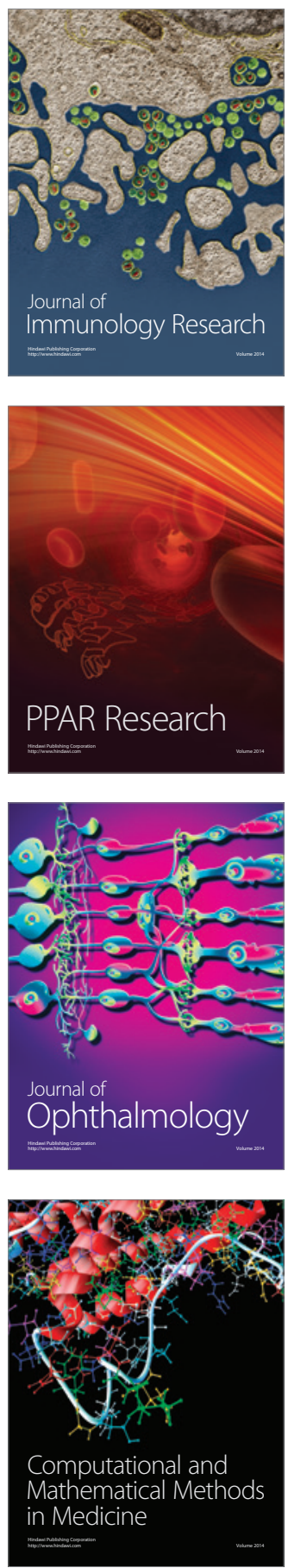

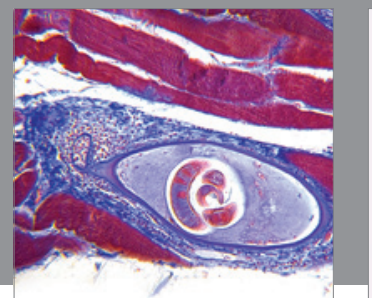

Gastroenterology

Research and Practice
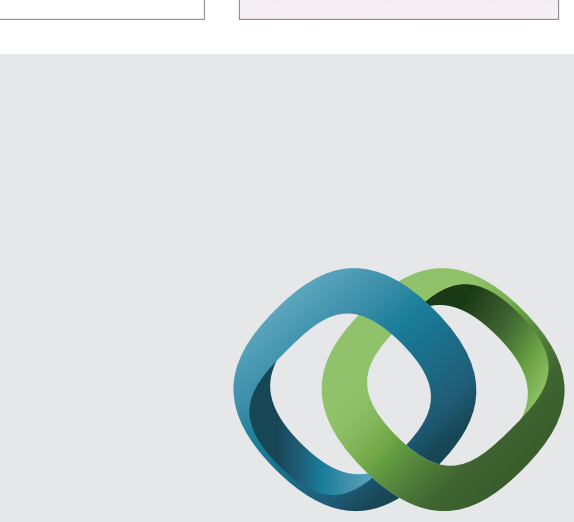

\section{Hindawi}

Submit your manuscripts at

http://www.hindawi.com
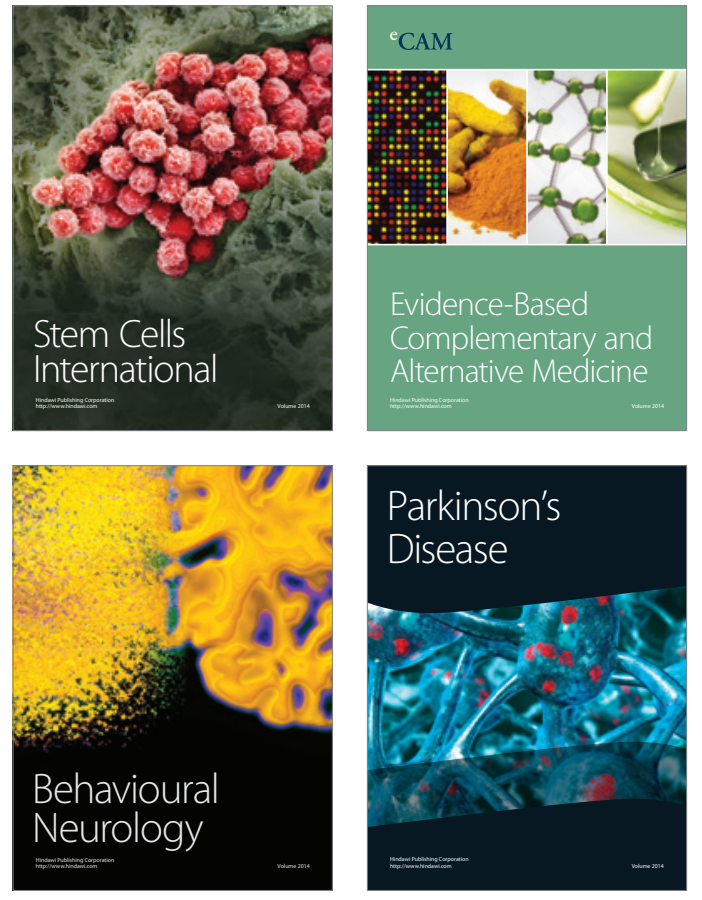
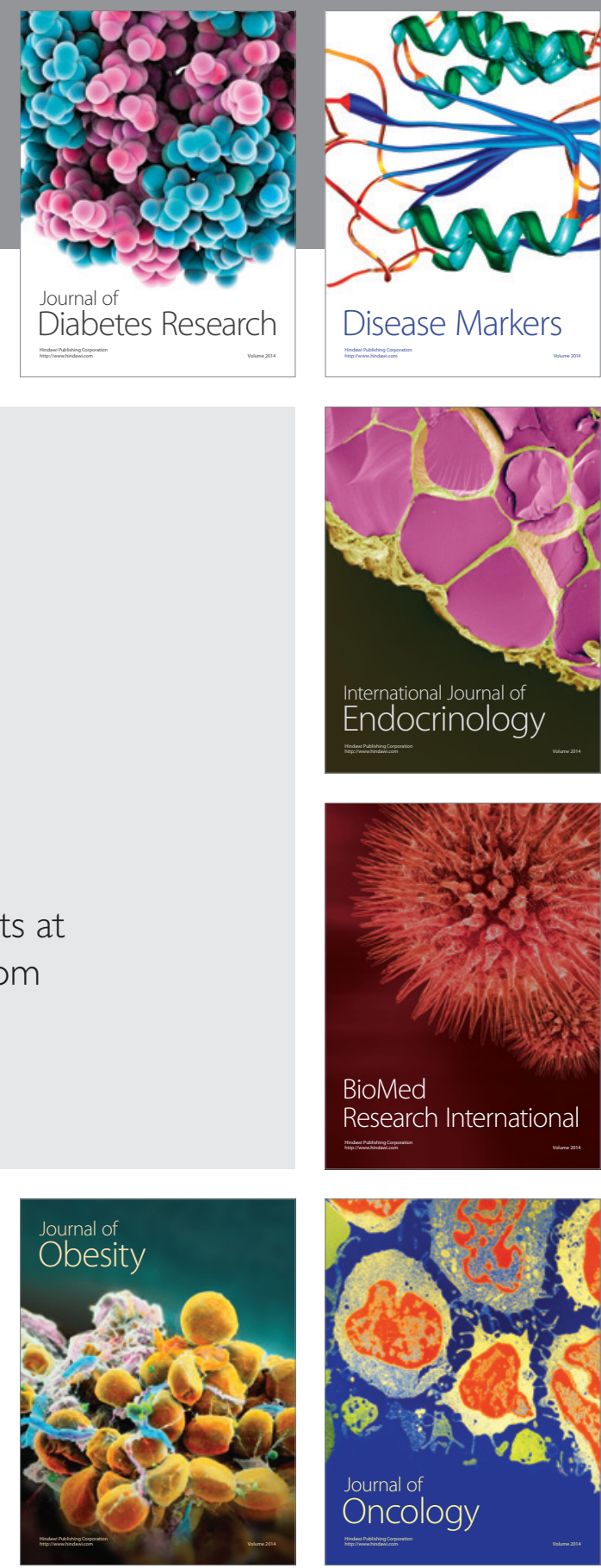

Disease Markers
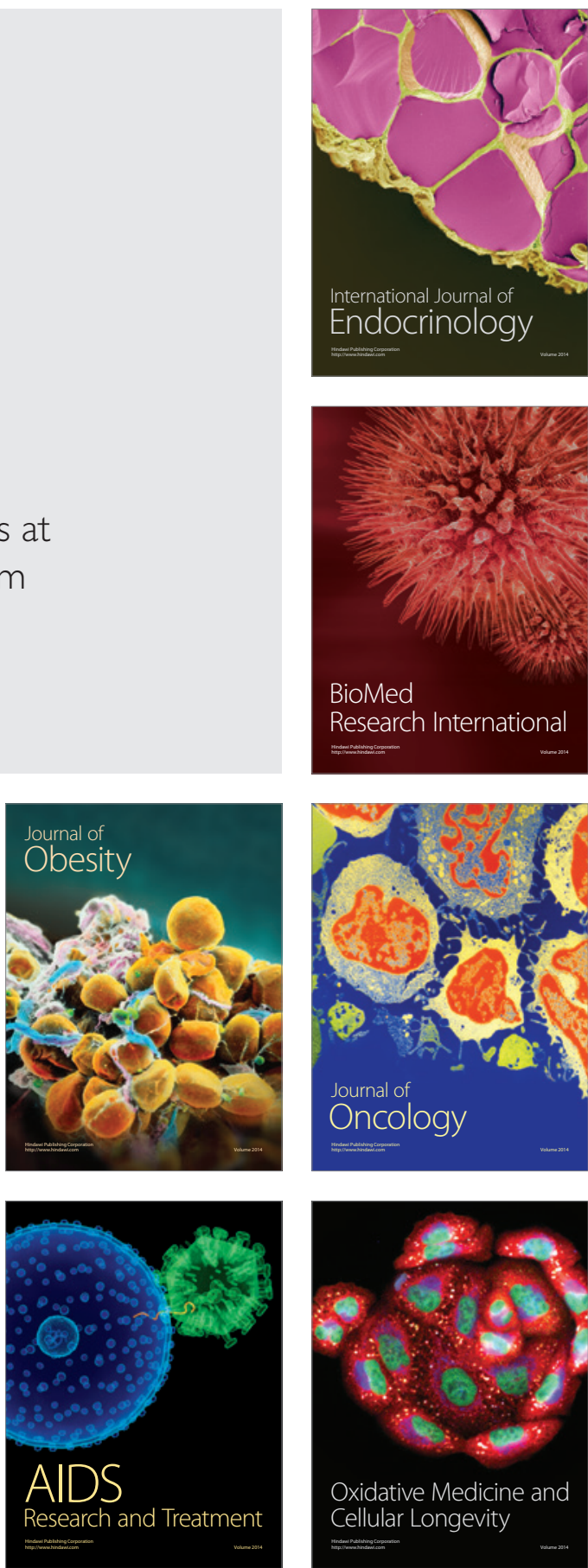